Results $51 \%$ of hospitalised IVH treated IBD patients met the WHO criteria of DM (CBG>11 mmol/L), while $20 \%$ and $6 \%$ had a CBG $>14 \mathrm{mmol} / \mathrm{l}$ and $>20 \mathrm{mmol} / \mathrm{l}$, respectively. 8 patients had pre-existing DM, which was confirmed by admission $\mathrm{HbA1c}$. RFR indicated disease severity score, duration of IVH, HbA1c and electrolyte imbalances (64\%) were best predictors of hyperglycaemia. $49 \%$ were started on or switched biological therapy during admissions. 55\% were discharged on prednisolone, $14 \%$ on budesonide and $34 \%$ on no GC. 48 patients had HbA1c checked at 3 month follow-up of which 4 were in the diabetic range. 1 was known DM with elevated CBG during admission whose insulin had been titrated, 2 had elevated $\mathrm{CBG}$ as inpatients with no prior DM discharged on gliclazide and insulin respectively and 1 was on long-term steroids for asthma who did not have CBG $>11.0 \mathrm{mmol} / \mathrm{L}$ as inpatient. 4 other patients discharged on gliclazide for steroid induced DM had documented repeat HbA1c recorded, which were all in the normal range.

Conclusions Our data demonstrates that hyperglycaemia is common in IVH treated inpatients, therefore CBG monitoring should be routine practice. Predictive modelling (RFR) identifies more severe disease activity, duration of IVH treatment and $\mathrm{HbA1c}$ as risk factors for hyperglycaemia. The importance of IVH duration suggests hyperglycaemia risk may be physician-modifiable. Alternative treatment strategies such as earlier introduction of biologics, rapid steroid taper and nutritional support could be used to minimise medication associated metabolic instability in high risk patients.

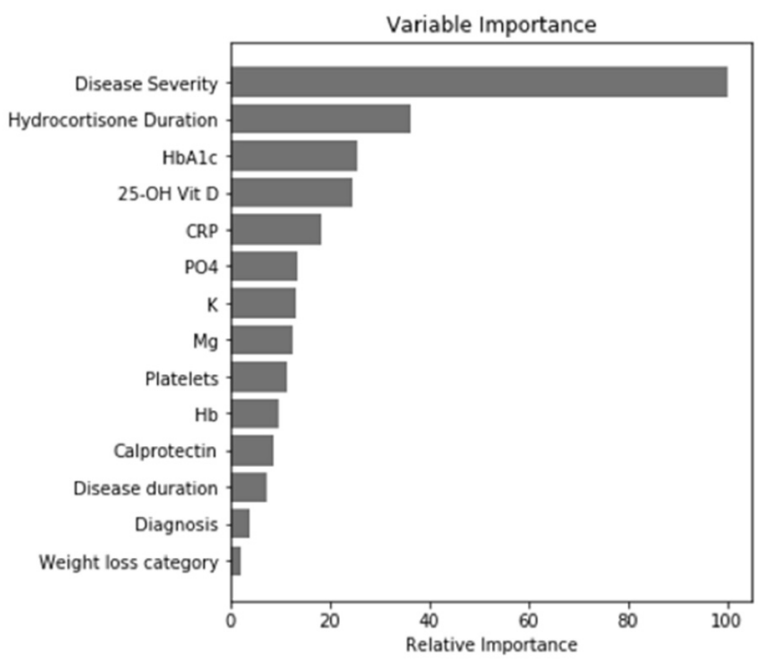

Abstract PTH 105 Figure 1 Relative importance of input features of RFR model for prediction of highest CBG

\section{PTH-106 PREDICTING RESPONSE TO VEDOLIZUMAB IN CROHN'S DISEASE - A SINGLE CENTRE EXPERIENCE}

${ }^{2}$ Claire Kane, ${ }^{1}$ Ross J Porter, ${ }^{2} J o h n$ Thomson, ${ }^{2}$ Malcolm Smith, ${ }^{2}$ Umesh Basavaraju, ${ }^{2}$ Gillian H Bain, ${ }^{1,2}$ Mairi H McLean*. ${ }^{1}$ University Of Aberdeen, Aberdeen, UK; ${ }^{2}$ Aberdeen Royal Infirmary, Aberdeen, UK

\subsection{6/gutjn--2019-BSGAbstracts. 165}

Introduction There is a clinical need to develop personalised clinical biomarker scoring systems to direct biologic treatment for Crohn's disease (CD). Vedolizumab is a humanised anti- $\alpha$ $4 \beta 7$ integrin biologic, inhibiting migration of lymphocytes to the gastrointestinal mucosa. Dulai and colleagues [Gastroenterology, 2018; 155(3)] developed a new clinical prediction tool to determine probability of response to Vedolizumab. Our aim was to retrospectively determine whether this published clinical prediction tool could identify responders to Vedolizumab in our single tertiary IBD centre.

Methods Patients receiving Vedolizumab for $\mathrm{CD}$ between 2015-2018 were identified from the IBD database. The Dulai clinical score $(>19$ predicting response and $\leq 13$ predicting non-response) was retrospectively calculated from baseline pretreatment characteristics including absence of previous antiTNF therapy $(+3)$, no previous gastrointestinal surgery $(+2)$, no fistulising disease $(+2)$ along with pre-treatment CRP (mg/ L) (variable) and albumin levels $(\mathrm{g} / \mathrm{L})$ (variable) as previously published. Clinical response at 26 weeks was determined based on documented improvement in clinical symptoms and no requirement for steroids. Radiological or endoscopic improvement in disease activity and reduction in faecal calprotectin were considered if data was available. Analysis was performed with SPSS V25 with Mann-Whitney U test, Fisher's exact test and ROC analysis, with significance $\mathrm{p}<0.05$.

Results Forty-nine patients with CD received Vedolizumab. 10 were excluded from analysis as they did not reach the 26week endpoint. Following 26 weeks of Vedolizumab treatment, 13/39 (33.3\%) CD patients had evidence of response. Responders versus non-responders had a median pre-treatment predictive score of 16.3 (8.2-20.8) and 14.1 (6.6-13.5), respectively $(\mathrm{p}=0.222)$. ROC analysis reported area under the curve of 0.621 (95\% CI $0.44-0.81)(\mathrm{p}=0.222)$. A score $>19$ had poor sensitivity [23.08\% (95\% CI 50.38,53.81)] but good specificity $[84.62 \% \quad(95 \%$ CI $65.13,95.64)]$ for predicting response to therapy. A score $\leq 13$ had poor sensitivity $[42.31 \%$ (95\% CI 22.35, 63.08)] but good specificity [84.62\% (95\% CI 54.55, 98.08)] for predicting non-response. There was no association between predictive score $>19$ or $\leq 13$ and patients who did $(p=0.666)$ or did not $(p=0.151)$ respond to Vedolizumab therapy, respectfully.

Conclusions In this retrospective single centre tertiary IBD centre analysis, the previously published Dulai clinical score did not predict response to Vedolizumab in CD. Future prospective analysis and consideration of a modified score including additional parameters is warranted.

\section{PTH-107 A UK NATIONAL SURVEY OF THERAPEUTIC DRUG MONITORING WITH ANTI-TNF MEDICATIONS IN IBD}

${ }^{1}$ Shadab Nayeemuddin*, ${ }^{1}$ Gaurav Nigam, ${ }^{2}$ Evan Kontopantelis, ${ }^{3,4} \mathrm{Bu}$ Hayee,

${ }^{1,2}$ Jimmy Limdi. 'Pennine Acute Hospitals NHS Trust, Manchester, UK; ${ }^{2}$ University of Manchester, Manchester, UK; ${ }^{3}$ Kings College Hospital, London, UK; ${ }^{4}$ Kings College London, London, UK

\subsection{6/gutjnl-2019-BSGAbstracts. 166}

Introduction A growing body of evidence supports use of therapeutic drug monitoring (TDM) in improving efficacy and cost-effectiveness of anti-TNF therapy in patients with inflammatory bowel disease (IBD), supported by AGA guidelines. ${ }^{1}$ Existing and evolving knowledge of TDM in clinical practice is less well understood. Our objective was to assess attitudes and barriers to TDM use with anti-TNF's in the UK.

Methods A 17-question survey was distributed to members of the British Society of Gastroenterology. Information on clinician characteristics, demographics, use and barriers towards 
implementing TDM with anti-TNF's was collected. Logistic regression was used to predict factors influencing TDM use.

Results 243 respondents participated $(51.6 \%$ male) of which 237 respondents met inclusion criteria; treating $>5$ IBD patients and at least 1 with an anti-TNF per month. Of the total respondents, $45 \%$ were Consultant Gastroenterologists (GI), 40\% IBD Nurse Specialists (CNS) and 15\% GI Specialist Registrars (SPR). Of these 237 respondents, TDM was used by $95.7 \%$ for secondary loss of response; $71.4 \%$ for primary non-response and $53.6 \%$ used TDM proactively. Barriers for TDM use were time lag in receiving results $(27.1 \%)$, lack of awareness of guidelines (15.6\%), and cost (11.9\%). Clinicians working at a teaching hospital were more likely to use TDM compared to a district hospital (OR 2.6, 95\% CI 0.71-9.8). IBD CNS and GI SPR used TDM more often, when compared to Consultant GI (OR 2.6, 95\% CI 0.69-10 \& OR 1.5, 95\% CI $0.3-7.2$ respectively). Clinicians practising for $>20$ years were more likely to check TDM than less experienced clinicians (OR 4.1, 95\% CI 0.4-41.8). Clinicians with large volume IBD practice ( $>50 \%$ IBD patients per month) were more likely to check TDM than those seeing fewer IBD patients (OR 45.6, 95\% CI 7.5-275). Proactive TDM was more likely to be used by clinicians working in a tertiary care setting (OR 2.25, 95\% CI 0.84-6.05), IBD CNS (OR 1.2, $95 \%$ CI $0.6-2.1$ ), clinicians managing large volume IBD practice (OR 10.8, 95\% CI 1.2-90) and clinicians with 5-9 years of experience in practice (OR 2.6 \& CI 1.04-6.42).

Conclusions Large volume IBD centres with more experience of treating IBD patients are more likely to employ treatmentoptimising strategies with TDM. Significant barriers to TDM implementation in the UK are time lag from test to result, lack of awareness of current guidelines and evolving knowledge, cost and less experience. Validation of point of care testing, lower cost assays, and wider dissemination of current evolving paradigms with updated recommendations may further optimise treatment with anti-TNF therapies.

\section{REFERENCE}

1. Feuerstein JD, et al. Gastroenterology 2017;153(3):827-834.

\section{PTH-108 THE HIDDEN BURDEN OF FAECAL INCONTINENCE IN ACTIVE AND QUIESCENT ULCERATIVE COLITIS: AN UNDERESTIMATED PROBLEM?}

${ }^{1}$ Gaurav B Nigam*, ${ }^{1}$ Jimmy K Limdi, ${ }^{2}$ Shaheen Hamdy, ${ }^{3}$ Dipesh H Vasant. ${ }^{1}$ Pennine Acute Hospitals; ${ }^{2}$ Salford Royal Foundation Trust; ${ }^{3}$ Manchester University Foundation Trust

\subsection{6/gutjnl-2019-BSGAbstracts.167}

Introduction Despite advances in Ulcerative Colitis (UC) therapies, many patients suffer refractory defaecatory symptoms in the absence of active inflammation. For this group, treatment remains challenging, with a paucity of research and limited therapeutic options. In this prospective, ongoing study, we aim to determine the prevalence of faecal incontinence (FI) in patients with quiescent UC.

Methods In a cross-sectional study, consecutive patients with UC attending Inflammatory Bowel Disease (IBD) clinics were invited to participate. Patients completed a series of validated questionnaires; including an IBD-specific FI questionnaire (ICIQ-IBD questionnaire), Hospital Anxiety and Depression
Scale (HADS), the Rome IV diagnostic questionnaire, and the IBD-control questionnaire. Participants were requested to return a Faecal Calprotectin (FCP) within 2 weeks of completing questionnaires. Quiescent UC was defined as IBD-control 8 score $\geq 13$ and IBD-control-VAS $\geq 85$, and/or FCP levels $\leq 250$ (where available, FCP data were used in preference to IBD-control to classify UC activity). Data were compared between active and quiescent groups using chi-square and non-parametric tests.

Results Overall, $n=97$ UC patients $(n=50$ males, mean age 48 (range 18-82) participated. ICIQ-IBD data revealed that most patients experience FI (84/97 (87\%) during 'relapses'. Interestingly, 58/97 (60\%) reported FI when in 'remission', and this group had higher median HADS depression $(\mathrm{P}=0.0002)$, poorer QoL scores $(\mathrm{P}<0.0001)$, and trend towards higher HADS anxiety $(\mathrm{P}=0.09)$ scores, compared to those without FI. Disease activity data (IBD-control and/or FCP) were available for all patients, and based on these 61/97 (63\%) had quiescent UC. The prevalence of FI based on ICIQ-IBD did not differ between those with active $(22 / 36,61 \%)$ and quiescent UC $(36 / 61,59 \%), P=N S$. In those with FI on ICIQ-IBD, median IBD-FI symptom scores, IBD-FI QoL scores and HADS (anxiety: $\mathrm{P}=0.47$, depression: $\mathrm{P}=0.18$ ) did not differ between disease activity groups. However, within the quiescent group, patients that met the more stringent Rome IV criteria for FI $(n=13)$ had higher median IBD-FI symptom scores $(\mathrm{P}=0.007)$ and HADS-depression scores $(\mathrm{P}=0.05)$, a trend to worse IBD-FI QoL $(\mathrm{P}=0.07)$, but similar HADS-anxiety $(\mathrm{P}=0.68)$.

Conclusion This study is one of the first to identify that regardless of disease activity, FI affects most patients with UC, detrimentally impacting patients' psychological wellbeing, impairing their QoL, and should therefore routinely be screened for in clinics. There is an urgent need for further research in the often neglected area of FI and quiescent disease.

\section{PTH-109 FATIGUE IN PATIENTS WITH CROHN'S DISEASE ON LONG TERM VITAMIN B12}

${ }^{1}$ Rupali Patel ${ }^{*}$, 'Eleanor Whittingham, ${ }^{2}$ Lesley Roberts, ${ }^{3}$ sukhdev Singh. ${ }^{1}$ University of Birmingham, birmingham, UK; ${ }^{2}$ University of Warwick, Warwick, UK; ${ }^{3}$ Good Hope Hospital, Birmingham, UK

\subsection{6/gutjnl-2019-BSGAbstracts. 168}

Introduction Some individuals with Crohn's Disease who take long term three monthly Vitamin B12 injections complain of lack of energy and tiredness prior to their next B12 injection. Some have persuaded their General Practitioners to give the B12 injection more frequently than 3 monthly.

Methods We wanted to determine if this was a common experience,and also if seen in other causes of B12 deficiency besides Crohn's Disease.

453 patients on long term B12 in the community were invited to complete a questionnaire, 148 agreed. Most of these patients were elderly with a history of pernicious anaemia. 445 patients completed the questionnaire online. Most of these patients had Crohn's disease. 593 questionnaires were analysed. 